\title{
Continuous and Simultaneous Multigas Monitoring Using a Highly Sensitive and Selective Photoacoustic Sensor
}

\author{
Stéphane Schilt, Jean-Philippe Besson and Luc Thévenaz \\ Nanophotonics and Metrology Laboratory, Swiss Federal Institute of Technology (EPFL), CH-1015 Lausanne, Switzerland \\ Stephane.Schilt@epfl.ch \\ Marcel Gyger \\ Center for the Study of Living Systems, Swiss Federal Institute of Technology (EPFL), CH-1015 Lausanne, Switzerland
}

\begin{abstract}
A multi-gas photoacoustic sensor is reported. Simultaneous measurement of ammonia, carbon dioxide and water vapor is demonstrated at ppm level using semiconductor lasers. In-situ measurements to control the housing conditions of laboratory animals are presented. (C)2004 Optical Society of America

OCIS codes: (300.6430) Spectroscopy, optoacoustic; (280.3420) Laser sensors.
\end{abstract}

\section{Introduction}

Photoacoustic spectroscopy (PAS) is an analytical technique widely recognized in trace gas detection for its high performances in term of sensitivity and selectivity. In addition to its simplicity, this technique also enables on-line and real-time measurement with a short response time and without sampling requirement. Whereas the technique has demonstrated its suitability for continuous gas monitoring at trace level (from ppm down to ppb levels) in various fields of applications, such as atmospheric researches [1], air-pollution measurements [2-3], industrial process control [3] or medical applications [4], it was most often applied to the detection of a single species.

We report in this paper the realization of a photoacoustic (PA) sensor capable of measuring simultaneously and continuously three different gases. The use of fiber-coupled distributed feedback (DFB) lasers enables a simple sensor architecture, as optical fibers largely facilitate the coupling of several lasers into one measurement PA cell. The performances of the sensor are illustrated by in-situ measurements performed in an animal house in order to analyze and control the evolution of the housing conditions of some laboratory rodents. For this purpose, the concentration of three physiological gases of interest, namely ammonia $\left(\mathrm{NH}_{3}\right)$, carbon dioxide $\left(\mathrm{CO}_{2}\right)$ and water vapor $\left(\mathrm{H}_{2} \mathrm{O}\right)$, has been recorded continuously into the atmosphere of individually ventilated cages housing mice, during a complete cycle of the bedding, in order to analyze the microclimate in the cages.

The concentration level of these gases is relevant for the wellbeing of the laboratory animals, an important contribution to the quality of in vivo research. Ammonia and water vapor reflect the quality of bedding and the ventilation rate inside the cage and thus, the level of hygiene. Carbon dioxide is an indicator of the physical activity of living organisms allowing assessment of the circadian activity of the mice in the cages.

\section{Sensor architecture}

Our multi-gas sensor is based on a resonant PA cell operated on its first longitudinal mode and injected by three different lasers. The sensor architecture is shown in Fig. 1(a). The PA cell is built out of stainless steel and consists in two large buffer volumes and a central cylindrical tube of radius $R_{c}=3 \mathrm{~mm}$ acting as an acoustic resonator [5]. The three lasers are fiber-coupled DFB laser diodes emitting in the optical telecommunication wavelength range, respectively at $1369 \mathrm{~nm}$ for $\mathrm{H}_{2} \mathrm{O}$ detection, at $1572 \mathrm{~nm}$ for $\mathrm{CO}_{2}$ and at $1532 \mathrm{~nm}$ for $\mathrm{NH}_{3}$. The emissions from the two first lasers are combined using a fiber coupler that is terminated with a beam collimator directly mounted on the outer flange of the buffer volume of the PA cell. The optical power is $23 \mathrm{~mW}$ for the $1572 \mathrm{~nm}$ laser $\left(\mathrm{CO}_{2}\right.$ detection) and $22 \mathrm{~mW}$ for the $1369 \mathrm{~nm}$ laser $\left(\mathrm{H}_{2} \mathrm{O}\right)$, but a very small fraction of this power is actually launched into the cell. The reason is the reduced absorption of the $\mathrm{CO}_{2}$ molecules in the spectral range of interest, so that the power from the laser for $\mathrm{CO}_{2}$ detection must be maximized in the PA cell using a strongly unbalanced directional coupler (see Fig. 1a). The resulting loss of power of the laser for water vapor monitoring does not have any consequence, because a detection limit in the $20 \mathrm{ppb}$ range is achievable by our sensor with the full laser power, whereas much higher concentrations (in the percent range) have to be measured in this particular application.

The fiber from the third laser $\left(\mathrm{NH}_{3}\right.$ detection) is also terminated with a beam collimator that is mounted on the outer flange of the other buffer volume of the PA cell, thus propagates in the opposite direction along the PA cell. 
This configuration enables to benefit from the entire optical power of this laser $(25 \mathrm{~mW})$, thus improving the detection limit of $\mathrm{NH}_{3}$, which is necessary to detect the low $\mathrm{NH}_{3}$ concentration encountered in this application.

(a)

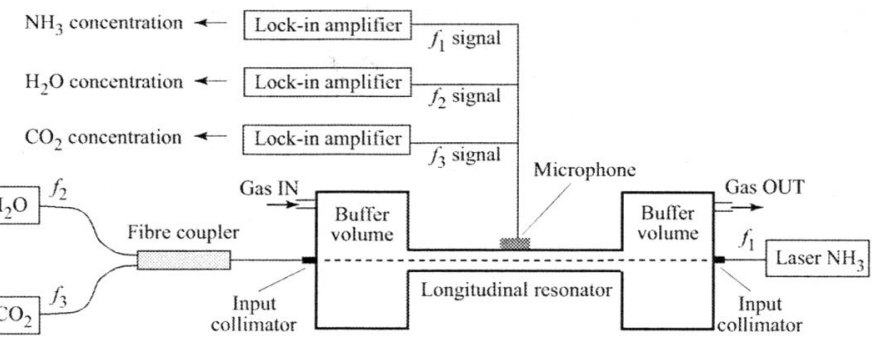

(b)

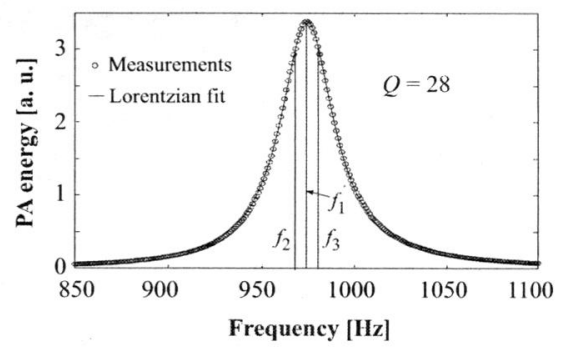

Fig. 1. (a) Schematic representation of the PA sensor based on three DFB lasers and a PA cell operated in its first longitudinal mode. The lasers are modulated at three different frequencies $f_{1}, f_{2}$ and $f_{3}$ and three lock-in amplifiers extract the corresponding concentrations from the microphone signal. (b) Acoustic resonance of the PA cell. Circles are experimental points and the curve is the result of a lorentzian fit. The three laser modulation frequencies are represented.

The three laser beams simultaneously propagate along the cell axis in order to efficiently excite the first acoustic longitudinal mode of the resonator. The sound waves generated as a result of molecular absorption are detected with a single electret microphone, located in the center of the resonator. In order to separate the acoustic signals generated by the different species, the lasers are modulated at three slightly different frequencies, all located in the resonance peak of the acoustic cavity, as shown in Fig. 1(b). The laser for $\mathrm{NH}_{3}$ detection is modulated at the resonant frequency $f_{1}$ in order to benefit from the largest acoustic amplification. An acoustic quality factor $Q=28$ is achieved in the resonator. The lasers for $\mathrm{H}_{2} \mathrm{O}$ and $\mathrm{CO}_{2}$ detection are modulated at frequencies $f_{2}=f_{1}-5 \mathrm{~Hz}$ and $f_{3}=f_{1}+5 \mathrm{~Hz}$, respectively. With so small differences between the three frequencies, the loss in the acoustic amplification is minor, whereas cross-talk between the corresponding acoustic signals is suppressed through highly selective lock-in detection at frequencies $f_{1}, f_{2}$ and $f_{3}$, respectively. This configuration enables the simultaneous and selective measurement of the three gases of interest. Finally, an electronic module controls the laser modulation and processes the data.

\section{Results}

The response of the sensor to various ammonia concentrations is shown in Fig. 2. The temperature of the laser was tuned to reach the appropriate absorption line and the laser was current-modulated with optimized amplitude to achieve the strongest PA signal. Different gas mixtures were generated from certified concentrations diluted using mass flow controllers. An excellent linearity is obtained with a detection limit of $0.2 \mathrm{ppm}$ of $\mathrm{NH}_{3}$ (defined for a signal-to-noise ratio $S N R=3$ ). A similar behavior has been achieved for $\mathrm{CO}_{2}$ and $\mathrm{H}_{2} \mathrm{O}$ (not shown here), with corresponding detection limits of $40 \mathrm{ppm}$ for $\mathrm{CO}_{2}$ and $8 \mathrm{ppm}$ for $\mathrm{H}_{2} \mathrm{O}$ in the experimental conditions of Fig. 1. For $\mathrm{H}_{2} \mathrm{O}$, the detection limit may potentially be reduced to $20 \mathrm{ppb}$ using the full laser power.
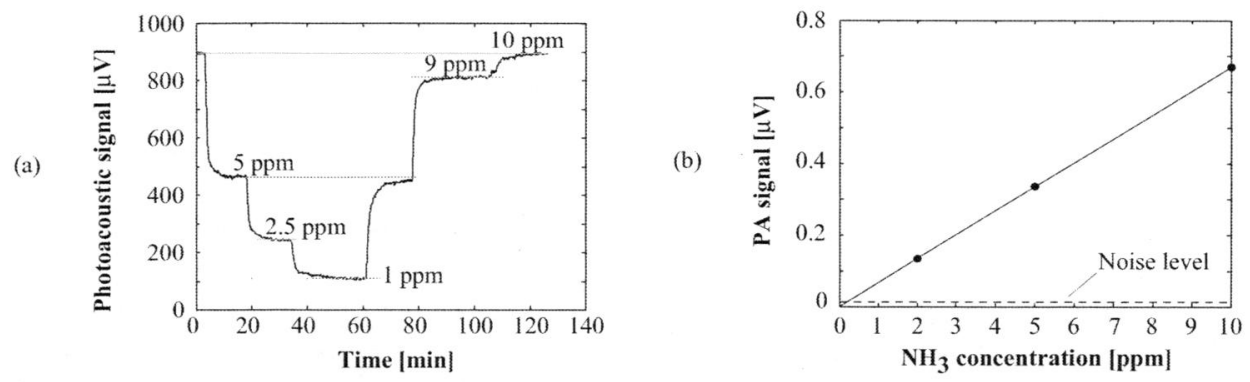

Fig. 2: (a) Response of the sensor to different $\mathrm{NH}_{3}$ concentrations; (b) $\mathrm{NH}_{3}$ calibration curve.

On-line and real time measurements of $\mathrm{NH}_{3}, \mathrm{H}_{2} \mathrm{O}$ and $\mathrm{CO}_{2}$ were performed during a period of two weeks (from Day 1 to Day 11) in individually ventilated cages housing laboratory mice. Fourteen cages containing a different number of animals, scaling from 1 adult to a couple with 11 pups, have been monitored during this period. For this purpose, air was pumped out of one of the cages into a Teflon tube at a flow rate of $1 \mathrm{l} / \mathrm{min}$. This flow rate results in a fast response time of the sensor without inducing additional acoustic noise. The gas passed through the PA cell and the concentration of the three gases was determined.

Ammonia concentration was monitored in the different cages on a half-week basis after the bedding cleaning. The average values are shown in Fig. 3(a). At Day 7, one cage already exceeds a concentration of 25 ppm, which 
corresponds to the human threshold limit value for this chemical substance in working environment [6]. After 11 days (date of the next bedding change), a second cage also presents a value over this limit and three others are about to reach it. A high level of ammonia, close to $70 \mathrm{ppm}$, has been observed in the dirtiest cage. This high level may be dangerous for animals' health if it is maintained during several days.

$\mathrm{CO}_{2}$ and $\mathrm{H}_{2} \mathrm{O}$ concentrations monitored continuously during three days in a selected cage are presented in Fig. 3(b). $\mathrm{CO}_{2}$ variations show a 12-hours time period, representing night and day activity. Correlation between water vapor and carbon dioxide cycles are also observed, showing an increase of perspiration of active animals. $\mathrm{CO}_{2}$ monitoring is also important as it enables the detection of accidental discontinued ventilation for which an increase of the $\mathrm{CO}_{2}$ concentration in the cages may be critical for animal's survival. $\mathrm{CO}_{2}$ concentrations monitored in cages disconnected from the ventilation have shown a drastic increase of $\mathrm{CO}_{2}$ immediately after the aeration interruption, reaching levels higher than $1.2 \%$.
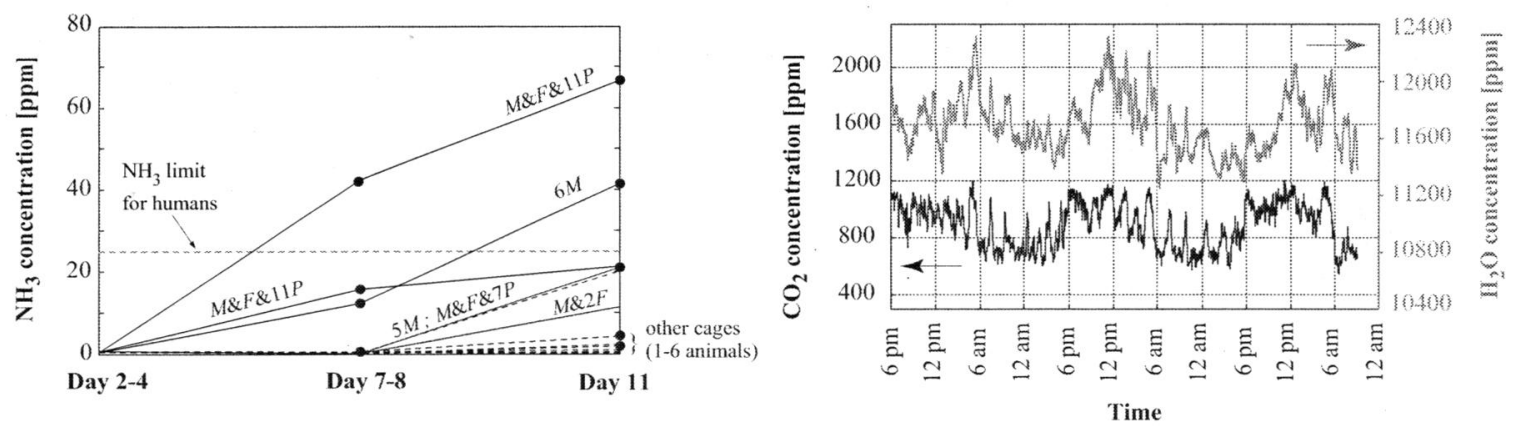

Fig. 3. (a) Evolution of ammonia concentration from Day 2 (after bedding change) to Day 11 in different cages. The content of the cages is indicated in the label of the curves; $M$ stands for male, $F$ for female and $P$ fur pups. (b) Evolution of $\mathrm{H}_{2} \mathrm{O}$ and $\mathrm{CO}_{2}$ levels in a selected cage (containing 6 males) from Day 4 to Day 7.

\section{Conclusion}

A multi-gas sensor has been developed based on resonant PAS. The sensor architecture has enabled a simultaneous and selective measurement of three different species, ammonia, carbon dioxide and water vapor in the present experiments, using three fiber-coupled DFB lasers. The number of analyzed species may even be increased by adding supplementary lasers at suitable wavelength and using proper modulation frequencies. In the present configuration, detection limits of $0.2 \mathrm{ppm} \mathrm{NH}_{3}, 40 \mathrm{ppm} \mathrm{CO}$ and $8 \mathrm{ppm} \mathrm{H}_{2} \mathrm{O}$ have been achieved (for $S N R=3$ ). The detection limit for $\mathrm{H}_{2} \mathrm{O}$ may even be reduced to $20 \mathrm{ppb}$ when using the full laser optical power.

In-situ application of the sensor has been demonstrated for the monitoring of microclimatic parameters in individually ventilated cages in an animal house. Continuous measurements have been successfully performed during two weeks and 24 hours a day without any maintenance of the sensor. Simultaneous measurement of $\mathrm{NH}_{3}$, $\mathrm{CO}_{2}$ and $\mathrm{H}_{2} \mathrm{O}$ has provided a better understanding of the evolution of the bedding conditions in the cages. These results fully demonstrate the suitability of the photoacoustic technique for this application, thanks to its high sensitivity and selectivity and unattended operation. Circadian activity level of animals can also be studied with this technique. These measurements were firstly made for validation purposes of our sensor. The promising results open future perspectives of this technique in various fields of application, including medical diagnostic and environment monitoring.

\section{References}

[1] M. Szakáll, Z. Bozóki, A. Mohácsi, A. Varga and G. Szabó, "Diode laser based photoacoustic water vapor detection system for atmospheric research", Applied Spectro. 58, 792-798 (2004).

[2] A. Thöny and M.W. Sigrist, "New developments in $\mathrm{CO}_{2}$-laser photoacoustic monitoring of trace gases", Infrared Phys. Technol. 36, 585-615 (1995).

[3] S. Schilt, L. Thévenaz, M. Niklès, L. Emmenegger and C. Hüglin, "Ammonia monitoring at trace level using photoacoustic spectroscopy in industrial and environmental applications", Spectrochim. Acta A 60, 3259-3268, (2004).

[4] L.R. Narasimhan, W. Gordon and C.K.M. Patel, "Correlation of breath ammonia with blood ures nitrogen and creatinine during hemodyalysis", PNAS 98, 4617-4621, (2001).

[5] J.-Ph. Besson, S. Schilt and L. Thévenaz, "Multi-gas sensing based on photoacoustic spectroscopy using tunable laser diodes", Spectrochim. Acta A 60, 3449-3456 (2004).

[6] M.R. Gamble and G. Clough, "Ammonia build-up in animal boxes and its effect on rat tracheal epithelium", Lab Animals 10, 93-104 (1976). 